\title{
Treatment of chronic patellar tendinopathy using an exercise program consisting of eccentric training and static stretching exercises combined with high intensity light therapy. A pilot study
}

\begin{abstract}
The aim of the present pilot trial was to investigate the effects an exercise program consisting of eccentric training and static stretching exercises combined with High Intensity Light Therapy (HILT) in patients with Chronic Patellar tendinopathy (CPT). Eight patients who had CPT participated in the present pilot trial. They received an exercise program consisting of eccentric training and static stretching exercises combined with HILT. All patients received an exercise programme five times per week for four weeks. Patients were treated with HILT for 3 sessions per week for 4 weeks, 12 sessions in total. Pain and function were evaluated using the VISA-P score at baseline, at the end of treatment (week 4), and one month (week 8) after the end of treatment. At week 4, there was a rise in VISA-P score of about 37 units. This rise is maintained at the one month follow-up. The results suggest that the combination of an exercise program with HILT is an adequate treatment for patients with CPT. Further research to establish the relative and absolute effectiveness of such a treatment approach is needed.
\end{abstract}

Volume 10 Issue 2 - 2018

\author{
Evangelos Nicolaou PT,' Stasinopoulos \\ Dimitrios,' Demetris Lamnisos ${ }^{2}$ \\ 'Chair person/Assiociate Professor, Physiotherapy, Director of \\ Cyprus Musculoskeletal and Sports Trauma Research Centre \\ (CYMUSTR EC), Cyprus \\ ${ }^{2}$ Assistant Professor, Biostatistics, Co-ordinator Master in Public \\ Health, Department of Health Sciences, School of Sciences, \\ Cyprus
}

Correspondence: Dimitrios stasinopoulos,Assistant Professor, Biostatistics, Co-ordinator Master in Public Health, Department of Health Sciences, School of Sciences, [a] 6, Diogenes Str. Engomi, PO Box 22006, 1516, Nicosia, Cyprus, Email D.Stassinopoulos@euc.ac.cy

Received: February 27, 2018| Published: April 19, 2018

\section{Introduction}

Chronic Patellar Tendinopathy (CPT) is a common clinical condition that is managed by physical therapists and is common among athletes and non-athletes alike. CPT is characterized by the absence of inflammatory cells and prostaglandins and an increased presence of fibroblasts and disorganized collagen. ${ }^{1}$ Therefore, this condition is not inflammatory as originally thought but is a degenerative one. The ideal term for clinical diagnosis is patellar tendinopathy because this term refers to the painful tendon without implying the pathology. ${ }^{2}$ Jumper's knee was the first diagnostic term used for this condition. However, this condition can occur in people who are not athletes and athletes who do not perform jumping in their sports. Thus, jumper's knee is not an appropriate term for clinical diagnosis. ${ }^{3}$ Patellar tendonitis is also an incorrect term for clinical diagnosis because the condition is not inflammatory but is degenerative as mentioned. The best diagnostic term may be the term patellar tendinosis because this term refers to the pathology of the tendon. ${ }^{4}$

Extrinsic factors such as inappropriate footwear, sport technique, training errors, and intrinsic factors such as muscle weakness and/ or inflexibility, and misalignment are the main factors that lead to $\mathrm{CPT}^{1}$. It is most commonly characterized by pain at the inferior pole of the patella; although pain can also be at the tibial attachment, in the attachment of the tendon to the superior pole of the patella as well as midsubstance pain has been reported ${ }^{3}$. The pain can be caused by performing a functional activity such as squat or hop. ${ }^{5}$

However, no ideal treatment has emerged for the management of CPT. Many clinicians advocates a conservative approach ${ }^{5}$ and physiotherapy is usually recommended. ${ }^{4} \mathrm{~A}$ wide array of physiotherapy treatments has been recommended for the management of CPT such as electrotherapeutic/physical modalities, exercise programmes, soft tissue manipulation, and manual techniques. ${ }^{1}$ These treatments have different theoretical mechanisms of action, but all have the same aim, to reduce pain and improve function. Such a variety of treatment options suggests that the optimal treatment strategy is not known, and more research is needed to discover the most effective treatment in patients with CPT.

One of the most common physiotherapy treatments for CPT is exercise. Eccentric exercise has shown good clinical results in $\mathrm{CPT}^{6}$ as well as in conditions similar to CPT in clinical behaviour and histopathological appearance, such as lateral elbow, ${ }^{7}$ rotator cuff ${ }^{8}$ and Achilles tendinopathy. ${ }^{6}$ Tightness of hamstring and quadriceps has been found in patients with patellar tendinopathy as an aetiological factor. ${ }^{9}$ Eccentric training of patellar tendon and static stretching exercises of quadriceps and hamstrings was an effective treatment approach in $\mathrm{CPT}^{2}$

Low-level laser therapy (LLLT) is the form of light therapy that is usually recommended as a supplement to the exercise program in the management of tendinopathies. ${ }^{10}$ More recently, physiotherapists have been able to use a new modality of light therapy for the management of tendinopathies, one known as High Intensity Laser Therapy (HILT). In HILT an Nd: YAG laser is employed. The laser has a wavelength of $1064 \mathrm{~nm}$ which causes minor and slow light absorption by chromophores and deliver radiation non-invasively to deep tissue to ensure treatment efficacy ${ }^{11}$. In addition, to having a higher power than low intensity lasers, laser used in HILT have a shorter laser emission 
time and a longer laser emission interval (low duty cycle). Therefore, a large amount of laser irradiation can be delivered to deep tissues. ${ }^{12}$

To our knowledge, there have been no studies to investigate the effectiveness of HILT as a supplement to an exercise program consisting of eccentric training and static stretching exercises in the management of tendinopathies. Therefore, the aim of the present pilot trial was to investigate the effects an exercise program consisting of eccentric training and static stretching exercises and HILT in patients with CPT.

\section{Materials and methods}

\section{Subjects}

Patients between 18 and 30 years old who were experiencing anterior knee pain were examined and evaluated in a private outpatient physiotherapy clinic located in Larnaka, Cyprus, between March 2016 and June 2016. All patients lived in Cyprus, were native speakers of Greek and were either self-referred or referred by their physician or physiotherapist. The selection criteria for the study were: ${ }^{5,13,14}$

A. Tenderness with palpation over the inferior pole of the patella;

B. No history of trauma to the knee;

C. Minimum duration of symptoms three months;

D. Unsuccessful conservative treatment before entering the study, but not in the preceding one month;

E. No other current knee or lower extremity problems including anterior knee pain, muscle strains and hip or ankle injuries;

F. Positive decline squat test. ${ }^{5,13,15}$ This is a clinical diagnostic test.

\section{Pain and functional capacity measurements}

The VISA-P questionnaire was used to monitor the pain and function of patients. The instrument is a simple questionnaire, consisted of eight questions that takes less than five minutes to complete and once patients are familiar with it they will be able to complete most of it themselves. It is a valid and reliable outcome measure for patients with patellar tendinopathy. ${ }^{16}$

\section{Dropout rate}

Reasons for patient dropout were categorized as follows: (1) withdrawal without reason, (2) not returned for follow-up, and (3) request for an alternative treatment.

\section{High-intensity laser therapy}

The technical characteristics of the HILT device (Figure 1) used in the present trial are presented in the Table 1. Two treatment protocols were used, one for analgesic effect and one for biostimulation effect (Table 2). The total area of HILT treatment was $25 \mathrm{~cm}^{2}$ according to the manufactures' instructions. HILT was applied to the most painful areas, firstly in a circular motion from the center toward the outside (analgesic effect) and secondly in a linear motion at the pain inflicting region (bio-stimulation effect).

\section{Procedure}

Two investigators were involved in the study: (1) the primary investigator who administered the treatments (EN); and (2) a lecturer (DS) who evaluated the patients to confirm the patellar tendinopathy diagnosis and performed all baseline and follow-up assessments, and gained informed consent. All assessments were conducted by DS who was blind to the patients' therapy group. DS interviewed each patient to ascertain baseline demographic and clinical characteristics, including patient name, sex, and age, duration of symptoms, previous treatment, occupation, a $\square$ ected leg and dominant leg.

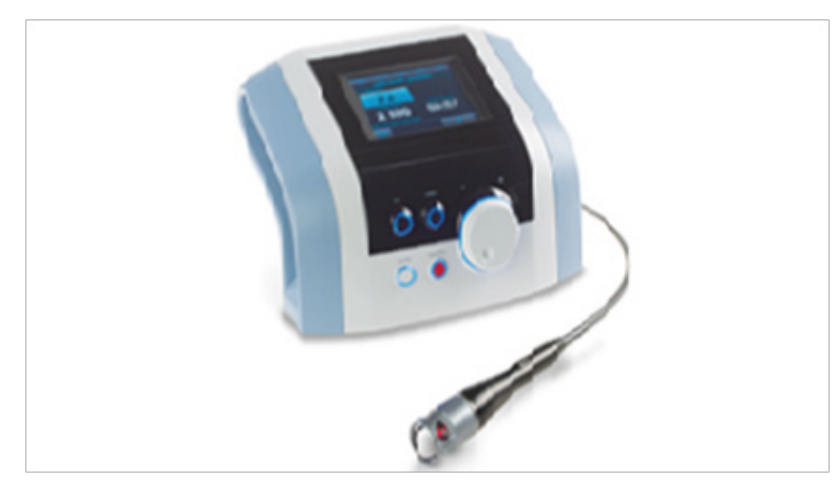

Figure I HILT device.

Table I Characteristics of HILT device

\begin{tabular}{ll}
\hline Total Output & I 2W, Continuous Mode \\
\hline $\begin{array}{l}\text { Operating wavelength } \\
\text { Mode of operation }\end{array}$ & I066 nm \\
Number of protocols & 38 \\
Safety Features & $\begin{array}{l}\text { Emergency off switch Operation by } \\
\text { footswitch Safety Interlock }\end{array}$ \\
Dimensions & $320 \times 190 \times 280 \mathrm{~mm}$ \\
Mains supply & $230 \mathrm{VI} 50-60 \mathrm{H}$ 'z, II $\mathrm{IV} / 50-60 \mathrm{~Hz}$ \\
Laser class & IV \\
$\begin{array}{l}\text { Equipment protection } \\
\text { class }\end{array}$ & IIB \\
\hline
\end{tabular}

Table 2 HILT protocol, analgesic and biostimulation effect

\begin{tabular}{lll}
\hline & Analgesic & Biostimulation \\
\hline Power $(\mathrm{W})$ & 9 & 5 \\
Dose $\left(\mathrm{J} / \mathrm{cm}^{2}\right)$ & $1 \mathrm{I}$ & 110 \\
Treatment area $\left(\mathrm{cm}^{2}\right)$ & 25 & 25 \\
Total Energy $(\mathrm{J})$ & 275 & 2750 \\
Treatment period $(\mathrm{min})$ & $2: 02$ & $9: 10$ \\
\hline
\end{tabular}

All patients received a written explanation of the trial before entry into the study and then gave signed consent to participate. The study was approved by the Cyprus Research Ethics Committee (EEBK/ $\mathrm{E} \Pi / 2016 / 05)$ and access to patients was authorized by the manager of the clinic (EN).

All patients were instructed to use their knee during the course of the study but to avoid activities that irritated the joint such as jumping, hopping and running. They were also told to refrain from taking anti-inflammatory drugs throughout the course of study. Patient compliance with this request was monitored using a treatment diary. 
Communication and interaction (verbal and non-verbal) between the therapist and patient was kept to a minimum, and behaviors sometimes used by therapists to facilitate positive treatment outcomes were purposefully avoided. For example, patients were given no indication of the potentially beneficial e $\square$ ects of the treatments or any feedback on their performance in the pre-application and postapplication measurements. ${ }^{17}$

Patients were treated with HILT as described above for 3 sessions per week for 4 weeks, 12 sessions in total. As eccentric exercises, participants carried out three sets of 15 repetitions of unilateral squat on a $25^{0}$ decline board. The squat was performed at a slow speed at every treatment session. The patients counted to 30 during the squat. ${ }^{15}$ As they moved from the standing to the squat position, the quadriceps muscle and patellar tendon by inference were loaded eccentrically; no following concentric loading was done, as the non-injured leg was used to get back to the start position. At the beginning, the load consisted of the body weight and participants were standing with all their body weight on the injured leg. Subjects were told to go ahead with the exercise even if they experienced mild pain. However, they were told to stop the exercise if the pain became disabling. When the squat was pain-free the load was increased by holding weights in their hands. Between each set there was a two-minute rest. Static stretching exercises of quadriceps and hamstrings were performed as described by Jensen \& Di Fabio ${ }^{18}$ before and after the eccentric training. ${ }^{19}$ Each stretch lasted 30seconds and there was a one minute rest between each stretch. Each training session was to be completed once daily, five times per week, for four weeks and was individualized on the basis of the patient's description of pain experienced during the procedure. ${ }^{18}$

\section{Statistical analysis}

Pain, function and dropout rate were measured in the present pilot trial. Each patient was evaluated at baseline (week 0), at the end of treatment (week 4) and one month (week 8) after the end of treatment. Data were analyzed using descriptive statistics.

\section{Results}

Fourteen patients eligible for inclusion visited the clinic within the trial period. Five did not meet the inclusion criteria described above. The other 9 patients were received the recommended treatment, an eccentric exercise program and HILT. There were 7 men and 2 women. The mean age (SD) of the sample was 30.90 (13.05) years and the duration of patellar tendinopathy were 5.573 months. Patellar tendinopathy was in the dominant leg in all patients.

The VISA-P was $56.67(22.14)$ at week 0 (Table 3). At week 4 , there was a rise in VISA score of about 37 units (Table 4). VISA P was 93.67 at week 8 (Table 3).

Table 3 VISA-P score before each evaluation

\begin{tabular}{llll}
\hline & Week 0 & Week 4 & Week 8 \\
\hline $\begin{array}{l}\text { Eccentric training } \\
\text { static stretching } \\
\text { and HILT }\end{array}$ & $56.67(22.14)$ & $94.00(10.39)$ & $93.67(10.97)$ \\
\hline
\end{tabular}

Values are means (SD).

There were no dropouts, no adverse e $\square$ ects were referred and all patients successfully completed the study.
Table 4 Change in VISA-P score

\begin{tabular}{ll}
\hline & $\begin{array}{l}\text { Eccentric training static } \\
\text { stretching and HILT }\end{array}$ \\
\hline Week 4 & $37.33($ I7.78) \\
Week 8 & $37.00(17.69)$
\end{tabular}

Values are means (SD)

\section{Discussion}

The present pilot study examined the effect of an exercise program consisting of eccentric training and static stretching exercises combined with HILT in the management of CPT. The findings of the present pilot trial have demonstrated significant improvements in terms of pain and disability. The results obtained from this pilot study are novel; as to date, similar studies have not been conducted.

Alfredson et al. ${ }^{20}$ first proposed the eccentric training of the injured tendon. It is the most commonly used conservative approach in the treatment of tendinopathy. Malliaras et al..$^{3}$ concluded that clinicians should consider eccentric-concentric loading alongside or instead of eccentric loading in Achilles and patellar tendinopathy. A Heavy Slow Resistance (HSR) program is recommended in the management of lower limb tendinopathy. ${ }^{21,22}$ The HSR program was produced equivalent pain and function improvement (VISA) than the Alfredson eccentric program, but significantly better patient satisfaction at six month follow-up. In the Achilles tendon, eccentric and HSR have recently been shown to yield similar clinical outcomes (VISA and patient satisfaction) at 1 year follow up. Based on the above findings, the HSR program can be recommended as an alternative to the Alfredson eccentric program lower limb tendinopathy rehabilitation.

Recently, isometric exercises have been recommended to reduce and manage tendon pain increasing the strength at the angle of contraction without producing inflammatory signs. ${ }^{3,23}$ Five repetitions of 45 -second isometric mid-range quadriceps exercise at $70 \%$ of maximal voluntary contraction have been shown to reduce patellar tendon pain for 45 minutes post exercise and this was also associated with a reduction in motor cortex inhibition of the quadriceps that was associated with patellar tendinopathy. ${ }^{23}$ The dosage of isometric contractions was based on clinical experience ${ }^{3,23,24}$ and their effect on pain in patients with CPT requires further study. The 'Spanish squat was used as isometric contraction and is useful when there is limited or no access to gym equipment as in the present situation. Therefore, it was hypothesized that the simultaneous use of these two kinds of contractions (isotonic and isometric) will further enhance the analgesic effect of contractions in the treatment of CPT, increasing the lower limb function.

A component lacking from evidence-based programs is adequate consideration of the kinetic chain. Poor lumbopelvic control has the potential to alter load distribution on the lower limb kinetic chain and increase the risk of lower limb tendinopathy. ${ }^{5,25}$ It is our belief that the improvement of lumbo-pelvic control can be achieved by performing simple exercises such as single leg bridging in supine and four point prone bridging exercises. Future research is needed to confirm this suggestion.

In addition, hip extensors weakness has been associated with patellar tendinopathy. ${ }^{26}$ Exercises to strengthen these muscle groups 
should be considered in exercise protocols and patellar tendinopathy. However, hip extensors were not strengthened in the present pilot trial. Functional activities such as jumping, cutting and sprinting should also be included in lower limb tendinopoathy rehabilitation programs among athletes, but have so far not been included in popular programs in the literature. ${ }^{26}$

The load of exercises was increased according to the patients symptoms otherwise the results are poor. ${ }^{18}$ Furthermore, eccentric exercises were performed at a low speed in every treatment session because this allows tissue healing. ${ }^{27}$ Ice was not recommended at the end of the treatment because research has shown that ice as a supplement to an eccentric exercise programme offers no benefit to patients with tendinopathy. ${ }^{28}$ Finally, the avoidance of painful activities is crucial for tendon healing, because training during the treatment period increases patients' symptoms and delays tendon healing. ${ }^{29}$

Eccentric exercises appear to reduce the pain and improve function. The mechanism by which eccentric training achieves these outcomes remains uncertain, as there is a lack of good quality evidence relating to physiological effects. The clinical improvement of the HSR group was accompanied by increased collagen turnover. It is unknown if the isometric contractions can reverse the pathology of the tendinopathy and in this case the pathology of CPT. Eccentric training with or without static stretching exercises is called an exercise programme. There are two types of exercise programme: home exercise programmes and exercise programmes carried out in a clinical setting which are called supervised exercise programmes. Although a home exercise programme can be performed any time during the day without requiring supervision from a therapist, our clinical experience has shown that patients fail to comply with the regimen of home exercise programmes..$^{30}$ Although many ways can be recommended to improve the compliance of patients with the home exercise programme such as phone calls, exercise monitors and better self-management education, it is believed that this problem can be solved by the supervised exercise programmes performed in a clinical setting under the supervision of a therapist. It is believed because our experience has shown that many patients stopped the home exercise programme without giving an explanation, whereas patients completed the supervised programme. One possible reason why they continue the supervised exercise programme could be the cost. In the supervised exercise programme, the patients visit the therapist more times than the home exercise programme, and this is more expensive. A future study will combine the both types of exercise programmes in order to maximize the compliance of the patients.

Although an exercise program is an effective treatment approach in a short period of time, a supplement to the exercise program should be found to reduce the treatment period. One such modality is LLLT, and research showed that LLLT plus an exercise program may be a better treatment approach than an exercise program alone for the management of LET ${ }^{31}$ and Achilles tendinopathy ${ }^{32}$ LLLT is a treatment that has been used for 26years in clinical practice ${ }^{33}$ for conditions such as tendinopathies, and it has been shown to have potential to modulate the degenerative process. As mentioned above, these tendon conditions are degenerative processes and not inflammatory. In addition, the biostimulatory effects of LLLT have been shown to reduce cell apoptosis ${ }^{34}$ and promote collagen fiber synthesis within a low-range therapeutic window of $0.4-4 \mathrm{~J}=\mathrm{cm} .{ }^{2,35,36}$
Like LLLT, HILT is also a light source, but LLLT and HILT differ in their radiation characteristics. It is claimed that HILT has biostimulative effects to accelerate cellular mechanisms and improve blood supply, but more research is needed to investigate precisely how HILT works, since there is little scientific evidence for the efficacy of HILT in musculoskeletal disorders, and absolutely no evidence of its efficacy for any other pathological condition. ${ }^{37}$ In addition, there are no guidelines on the parameters (dose, frequency, duration) of HILT. ${ }^{38}$ Finally, HILT has used in only one study in the management of tendinopathy, especially in LET with positive results. ${ }^{37}$

Even though the positive effects of such a therapeutic approach in CPT have been reported in the present pilot trial, its study design (small study population, no control, no comparison with other treatment modalities) limits the generalization of these findings. Future well-designed clinical trials are needed to confirm the positive results of this pilot study establishing the effectiveness of such an exercise program combined with HILT in the management of CPT. In addition, structural changes in the tendons related to the treatment interventions and the long-term effects (6 months or more after the end of treatment) of these treatments are needed to investigate. Further research is needed to establish the possible mechanism of action of this treatment approach, and the cost effectiveness of such treatment, because reduced cost is an important issue for the recommendation of any given treatment.

\section{Conclusion}

An exercise program consisting of eccentric and static stretching exercises, combined with HILT is an effective treatment approach for patients with CPT. Controlled studies are needed to establish the effects and the mechanism of action of such a treatment approach in CPT. A cost-effectiveness analysis should be incorporated into the analysis of the effectiveness of such a treatment approach in future trials.

\section{Acknowledgements}

None.

\section{Conflict of interest}

Authors declare there is no conflict in publishing the article.

\section{References}

1. Stasinopoulos D. Patellar Tendinopathy May not Be the Proper Term for Patients With Clinical Diagnosis of Patellar Tendon Disorder. Trauma Mon. 2014;19(2):e15301.

2. Dimitrios S, Pantelis M, Kalliopi S. Comparing the effects of eccentric training with eccentric training and static stretching exercises in the treatment of patellar tendinopathy. A controlled clinical trial. Clin Rehabil. 2012;26(5):423-30.

3. Malliaras P, Cook J, Purdam C, et al. Patellar Tendinopathy: Clinical Diagnosis, Load Management, and Advice for Challenging Case Presentations. J Orthop Sports Phys Ther. 45(11):887-98.

4. Peers KH, Lysens RJ. Patellar tendinopathy in athletes: current diagnostic and therapeutic recommendations. Sports Med 2005;35(1):71-87.

5. Kountouris A, Cook J. Rehabilitation of Achilles and patellar tendinopathies. Best Pract Res Clin Rheumatol. 2007;21(2):295-316. 
6. Malliaras P, Barton C, Reeves N, et al. Achilles and patellar tendinopathy loading programmes. a systematic review comparing clinical outcomes and identifying potential mechanisms for effectiveness. Sports Med. 2013;43(4):267-86

7. Raman J, MacDermid JC, Grewal R. Effectiveness of different methods of resistance exercises in lateral epicondylosis - a systematic review. J Hand Ther 2012;25(1):5-25; quiz 26.

8. Camargo PR, Alburquerque-Sendín F, Salvini TF. Eccentric training as a new approach for rotator cuff tendinopathy: Review and perspectives. World J Orthop. 2014;5(5):634-44.

9. Scattone Silva R, Nakagawa TH, Ferreira AL, et al. Lower limb strength and flexibility in athletes with and without patellar tendinopathy. Physical Therapy in Sport. 2016;20:19-25.

10. Bjordal J, Couppe C, Ljunggreen A. Low level laser therapy for tendinopathy: evidence of a dose-response pattern. Phys Ther Rev. 2001;6(2):91-99.

11. Basford JR. Low intensity laser therapy: still not an established clinical tool. Lasers Surg Med. 1995;16(4):331-42.

12. Santamato A, Solfrizzi V, Panza F, et al. Short-term effects of highintensity laser therapy versus ultrasound therapy in the treatment of people with subacromial impingement syndrome: a randomized clinical trial. Phys Ther. 2009;89(7):643-52.

13. Khan K, Cook J, Taunton J, et al. Overuse tendinosis, not tendonitis part 1: a new paradigm for a difficult clinical problem. The Physician Sportsmed. 2000;28(5):38-48.

14. Khan K, Maffulli N, Coleman D, et al. Patellar tendinopathy: Some aspects of basic science and clinical management. $\mathrm{Br} J$ Sports Med.1998;32(4):346-55.

15. Stasinopoulos D, Stasinopoulos I. Comparison of effects of exercise programme, pulsed ultrasound and transverse friction in the treatment of chronic patellar tendinopathy. Clin Rehabil. 2004;18(4):347-52.

16. Visentini PJ, Khan K, Cook J, et al. The Visa score: An index of severity of symptoms in patients with jumper's knee (patellar tendinosis). Victorian institute of sport tendon study group. J Sci Med Sport. $1998 ; 1(1): 22-8$

17. Vicenzino B, Collins, Wright A. The initials effects of a cervical spine manipulative physiotherapy treatment on the pain and dysfunction of lateral epicondylalgia. Pain. 1996;68(1):69-74.

18. Jensen K, Di Fabio R. Evaluation of eccentric exercise in treatment of patellar tendinitis. Phys Ther. 1989;69(3):211-6.

19. Purdam CR, Johnsson P, Alfredson $\mathrm{H}$, et al. A pilot study of the eccentric decline squat in the management of painful chronic patellar tendinopathy. Br J Sports Med. 2004;38(4):395-7.

20. Alfredson H, Pietilä T, Jonsson P, et al. Heavy-load eccentric calf muscle training for the treatment of chronic Achilles tendinosis. Am J Sports Med. 1998;26(3):360-6.

21. Beyer R, Kongsgaard M, Hougs Kjær B, et al. Heavy Slow Resistance Versus Eccentric Training as Treatment for Achilles Tendinopathy: A Randomized Controlled Trial. Am J Sports Med. 2015;43(7):1704-11.

22. Kongsgaard M, Kovanen V, Aagaard P, et al. Corticosteroid injections, eccentric decline squat training and heavy slow resistance training in patellar tendinopathy. Scand J Med Sci Sports. 2009;19(6):790-802.
23. Rio E, Kidgell D, Purdam C, et al. Isometric exercise induces analgesia and reduces inhibition in patellar tendinopathy. $\mathrm{Br} J$ Sports Med. 2015;49(19):1277-83.

24. Stasinopoulos D. The Effectiveness of Isometric Contractions Combined with Eccentric Contractions and Stretching Exercises on Pain and Disability in Lateral Elbow Tendinopathy. A case Report. $J$ Novel Physiother. 2015;5:238

25. Dimitrios S. Achilles Tendinopathy and Lumbopelvic Stability. Rheumatology. 2013;4:126.

26. Silva RS, Ferreira AL, Nakagawa TH, et al. Rehabilitation of Patellar Tendinopathy Using Hip Extensors Strengthening and Landing Strategy Modification: Case Report With 6-Months Follow-Up. J Orthop Sports Phys Ther. 2015;45(11):899-909.

27. Kraushaar B, Nirschl R. Tendinosis of the elbow (tennis elbow). Clinical features and findings of histological immunohistochemical and electron microscopy studies. J Bone Joint Surg Am. 1999;81(2):259-78.

28. Manias P, Stasinopoulos D. A controlled clinical pilot trial to study the effectiveness of ice as a supplement to the exercise programme for the management of lateral elbow tendinopathy. $\mathrm{Br} J$ Sports Med. 2006;40(1):81-5.

29. Visnes H, Hoksrud A, Cook J, et al. No effect of eccentric training on jumper's knee in volleyball players during the competitive season: a randomized clinical trial. Clin J Sport Med. 2005;15(4):227-34.

30. Stasinopoulos D, Stasinopoulos I. Comparison of effects of Cyriax physiotherapy, a supervised exercise and polarized polychromatic noncoherent light (Bioptron light) for the treatment of lateral epicondylitis Clin Rehab. 2006;20(1):12-23

31. Stergioulas, A. Effects of low-level laser and plyometric exercises in the treatment of lateral epicondylitis. Photomed. Laser Surg. 2007;25(3):205-13

32. Stergioulas A, Stergioulas M, Aarskog R, et al. Effects of low-level laser therapy and eccentric exercises in the treatment of recreational athletes with chronic achilles tendinopathy. Am J Sports Med. 2008;36(5):881-7.

33. Goldman JA. Investigative studies of laser technology in rheumatology and immunology. Biomedical Laser Technology Clinical Applications. New York: Springer Verlag. 1981.

34. Carnevalli CM, Soares CP, Zangaro RA, et al. Laser light prevents apoptosis in Cho K-1cell line. J Clin Laser Med Surg. 2003;21(4):193-

35. Loevschall H, Arenholt-Bindslev D. Effect of low level diode laser irradiation of human oral mucosa fibroblasts in vitro. Lasers Surg Med. $1994 ; 14: 347-354$

36. Reddy GK, Stehno-Bittel L, Enwemeka CS. Laser photostimulation of collagen production in healing rabbit Achilles tendons. Lasers Surg Med. 1998;22:281-287.

37. Kim SH, Kim YH, Lee HR, et al. Short-term effects of high-intensity laser therapy on frozen shoulder: A prospective randomized contro study. Man Ther. 2015;20(6):751-7.

38. Akkurt E, Kucuksen S, Y1lmaz H, et al. Long term effects of high intensity laser therapy in lateral epicondylitis patients. Lasers Med Sci. 2016;31(2):249-53. 